\title{
Low penalty, dual stage, broadband discrete Raman amplifier for high capacity WDM metro networks
}

\author{
Lukasz Krzczanowicz, Md Asif Iqbal, Ian Phillips, Mingming Tan, Pavel Skvortcov, Paul Harper and \\ Wladek Forysiak \\ Aston Institute of Photonics Technologies, Aston University, Birmingham B4 7ET, UK \\ Authore-mail address: l.krzczanowicz@aston.ac.uk
}

\begin{abstract}
We present a broadband (>70nm), dual stage, discrete Raman amplifier built with small and standard core fibre with $\sim 19.5 \mathrm{~dB}$ net gain. We transmit $120 \mathrm{~Gb} / \mathrm{s}$ DP-QPSK signals over $3040 \mathrm{~km}$ with 38 amplifications for a preFEC BER $<3.8 \times 10^{-3}$.

OCIS codes: (190.5650) Raman effect; (140.3550) Lasers, Raman; (250.4480) Optical amplifiers.
\end{abstract}

\section{Introduction}

One convenient way of increasing network capacity is to enhance the optical transmission bandwidth around the presently used spectrum. Most installed fibre links are currently dominated by $\mathrm{C}$ band erbium doped fibre amplifiers (EDFAs), whose gain bandwidth is limited to $\sim 40 \mathrm{~nm}$. It is possible to combine EDFAs operating in different bands to extend the transmission bandwidth, but this technique generates a "bandwidth gap" and introduces excess losses due to band splitters. This can be easily avoided by using Raman amplification to provide seamless broad bandwidth gain [1].

Raman amplifiers can in principle have fully configurable gain spectra determined by the Raman gain spectra of multiple pump lasers and scale to more than $100 \mathrm{~nm}$ total bandwidth [2]. However, achieving a flat gain spectrum can be costly as it requires pumping at many wavelengths [3]. The majority of Raman amplifiers uses distributed amplification, where the transmission fibre itself is used for amplification, which improves the optical signal to noise ratio (OSNR) thereby extending transmission reach [1]. However, this method requires high optical pump powers to be launched into the transmission fibre, which can be disadvantageous for operational reasons, such as laser safety. An alternative is discrete Raman amplification, which uses a separate fibre as the gain medium. In choosing the right fibre type the effective area is an important factor because while a smaller core results in higher gain, it can also lead to increased nonlinear transmission penalties. Most reported discrete Raman amplifiers use dispersion compensating fibre (DCF) as the gain medium, which offer simultaneous compensation of loss and dispersion [4]. Other types of Raman gain fibre: highly nonlinear fibre (HNLF), dispersion shifted fibre (DSF) and inverse dispersion fibre (IDF) have also been investigated [5]. Apart from nonlinearity, the challenges of using these fibres include double Rayleigh scattering (DRS) and induced multipath interference (MPI). In particular, DRS significantly deteriorates the OSNR performance and sets an upper limit to the maximum gain of the amplifier of 10-15 dB, depending on the tolerable penalty per amplification [1]. As the DRS induced MPI noise tends to increase with the fibre length, it was found that using multiple gain stages with an optical isolator in between them reduces this effect and results in better overall performance [6].

In this paper we experimentally investigate the performance of a $>70 \mathrm{~nm}, 19.5 \mathrm{~dB}$ net gain with $<2.5 \mathrm{~dB}$ gain flatness dual stage amplifier built with IDF and SMF. The first stage consists of $10 \mathrm{~km}$ of IDF and provides $14.5 \mathrm{~dB}$ gain, while a $10 \mathrm{~km} \mathrm{SMF}$ second stage was designed to extend the gain by $5 \mathrm{~dB}$ with minimum additional nonlinear penalty due to its larger core area. To test the amplifier in long distance transmission, we measured the signal quality $\left(\mathrm{Q}^{2}\right)$ of amplified $120 \mathrm{~Gb} / \mathrm{s}$ DP-QPSK modulated signals in a recirculating loop, where we reach a distance of $38 \times 80=3040 \mathrm{~km}$ for a maximum pre-FEC BER of $3.8 \times 10^{-3}\left(\mathrm{Q}^{2}=8.5 \mathrm{~dB}\right)$.

\section{Amplifier design}

The discrete Raman amplifier design, gain, noise figure and the pump powers used are shown in Fig 1. It is made of two independent amplification stages, each backward pumped with $14 \mathrm{xx}$ semiconductor lasers. The first stage, consisting of $10 \mathrm{~km}$ IDF $\left(0.23 \mathrm{~dB} / \mathrm{km}\right.$ attenuation, $-44 \mathrm{ps} / \mathrm{nm}-\mathrm{km}$ dispersion and $31 \mu \mathrm{m}^{2}$ effective area at $\left.1550 \mathrm{~nm}\right)$, generated an average gain of $14.5 \mathrm{~dB}$ across $>70 \mathrm{~nm}$ from $1530 \mathrm{~nm}$ to $1600 \mathrm{~nm}$. We chose IDF for the first stage because of its improved balance between the gain efficiency and nonlinear penalties, having a core area greater than DCF it generates less nonlinear penalty while optimally utilizing our available pumping power. As shown in Fig. 1d, the first stage had a noise figure (NF) between $7 \mathrm{~dB}$ and $5.5 \mathrm{~dB}$, decreasing with wavelength. An additional $10 \mathrm{~km}$ of SMF extended the gain and output power, giving a total net gain of $19.5 \mathrm{~dB}$ with $<2.5 \mathrm{~dB}$ gain ripple and a small increase in the NF of $<0.3 \mathrm{~dB}$. 

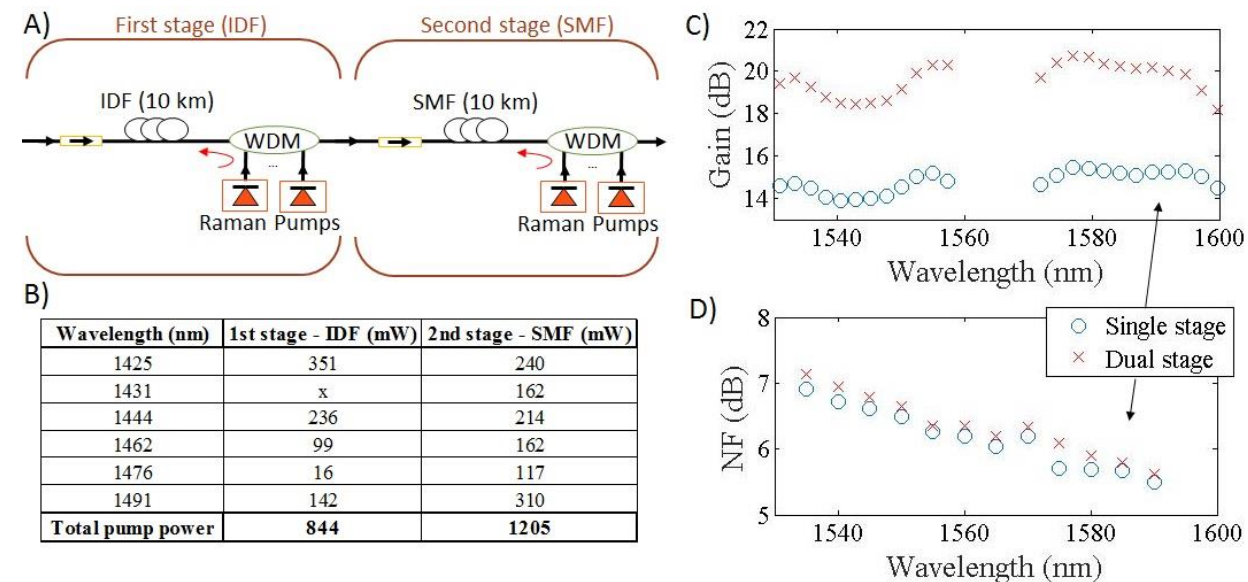

Fig. 1. a) Amplifier design, b) pump powers used, c) gain and d) noise figure.

\section{Broadband transmission setup and operating procedure}

The experimental transmission setup is shown in Fig. 2. For convenience, the transmitter consisted of $\mathrm{C}$ and $\mathrm{L}$ band EDFAs, whose ASE was shaped with the use of wavelength selective switches (WSS) to generate 24 channels with $300 \mathrm{GHz}$ spacing (12 in each band) from $195.8 \mathrm{THz}(1531.12 \mathrm{~nm})$ to $192.5 \mathrm{THz}(1557.36 \mathrm{~nm})$ in $\mathrm{C}$ band and $190.7 \mathrm{THz}(1572.06 \mathrm{~nm})$ to $187.4 \mathrm{THz}$ in $\mathrm{L}$ band $(1599.75 \mathrm{~nm})$. It has been previously shown that using channelized ASE is suitable for emulating nonlinear transmission performance in QPSK systems [7]. These were combined with a $100 \mathrm{kHz}$ linewidth tunable laser through a 50/50 coupler, which was used as a "channel under test" while the corresponding ASE channel was switched off during the measurement. A $120 \mathrm{Gbit} / \mathrm{s}$ DP-QPSK signal was generated using an IQ modulator and a polarization multiplexer (PolMux). The generated transmission spectrum was amplified with a pair of $\mathrm{C}$ and $\mathrm{L}$ band EDFAs before launch into a recirculating loop through an acousto-optic modulator (AOM).

The loop itself consisted of a $59 \mathrm{~km}$ SMF transmission span ( $12.5 \mathrm{~dB}$ loss) for measurements using the single stage Raman amplifier and a $80 \mathrm{~km}$ SMF span (17 dB loss) in the case of the dual stage amplifier. The amplifier was followed by a C/L band splitter. The $\mathrm{C}$ band part of the spectrum was gain flattened by a gain flattening filter (GFF) whereas a WSS was used to flatten the $\mathrm{L}$ band due to the lack of a broadband leveler. This was followed by $\mathrm{C}$ and $\mathrm{L}$ band EDFAs to compensate for the additional loop losses $(\sim 14 \mathrm{~dB})$ generated by GFFs, couplers and AOM.

The receiver chain consisted of a tunable band pass filter (BPF) followed by an EDFA to provide constant power into a polarization diverse coherent receiver, where the signal was mixed with a $100 \mathrm{kHz}$ linewidth local oscillator and captured using an $80 \mathrm{GSa} / \mathrm{s}, 36 \mathrm{GHz}$ real-time oscilloscope. The recorded data was then processed using offline Digital Signal Processing (DSP), where the $\mathrm{Q}^{2}$ was derived from the bit error ratio (BER) [8].

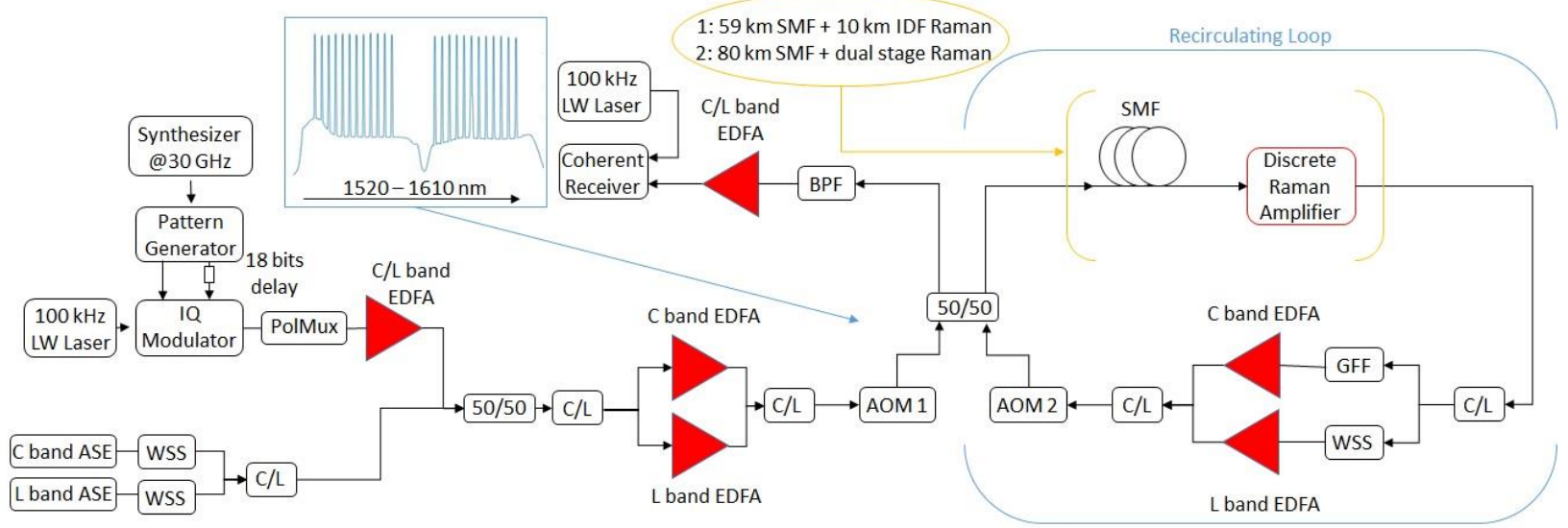

Fig. 2. Broadband transmission setup.

\section{Transmission results and discussion}

Fig. 3a. shows the $\mathrm{Q}^{2}$ vs distance for the middle channel of both bands $(1545.32 \mathrm{~nm}$ and $1584.53 \mathrm{~nm}$ ) for the single stage Raman amplifier compensating for the $59 \mathrm{~km} \mathrm{SMF}$ transmission span and Fig. 3b. shows the dual stage Raman amplifier compensating for the $80 \mathrm{~km}$ SMF span in the recirculating loop. We assumed a $\mathrm{Q}^{2}$ threshold of $8.5 \mathrm{~dB}$ 
(BER 3.8 $\times 10^{-3}$ ) for forward error correction (FEC). As anticipated, due to increased span loss, the $80 \mathrm{~km}$ configuration compensated by the dual stage amplifier has a shorter overall reach when compared to the $59 \mathrm{~km}$ configuration compensated by a single stage amplifier. In both cases the transmission in $\mathrm{L}$ band showed higher $\mathrm{Q}^{2}$ than in the $\mathrm{C}$ band due to the NF tilt. Fig $3 \mathrm{c}$. shows $\mathrm{Q}^{2}$ for selected channels at a distance of $3040 \mathrm{~km}$ with the use of the dual stage Raman amplifier, the distance achieved throughout the $\mathrm{C}$ and $\mathrm{L}$ band. We were not able to test the channels above $1590 \mathrm{~nm}$ (or in the gap between $\mathrm{C}$ and $\mathrm{L}$ bands) because of equipment limitations, but the NF trend suggests the $\mathrm{Q}^{2}$ should be above the FEC threshold in that region. The difference in the noise floor between $\mathrm{C}$ and $\mathrm{L}$ band output spectra (shown in inset of Fig. 3c.) is due to the different devices used for gain flattening of each band.

While the long distance transmission demonstrated here confirms the low penalty due to intra-channel nonlinearity of these discrete Raman amplifiers, it is in short distance metro networks where capacity growth is currently greatest and we anticipate using higher order modulation formats such as DP-64-QAM in the near future [9]. Recent improvements in SOAs [10] could offer comparably high bandwidth in metro networks and in data centre applications, but the nonlinear penalty due to repeated SOA passes is still an issue [11] while the proven cascadability of discrete Raman amplifiers could make them an attractive choice for such links.
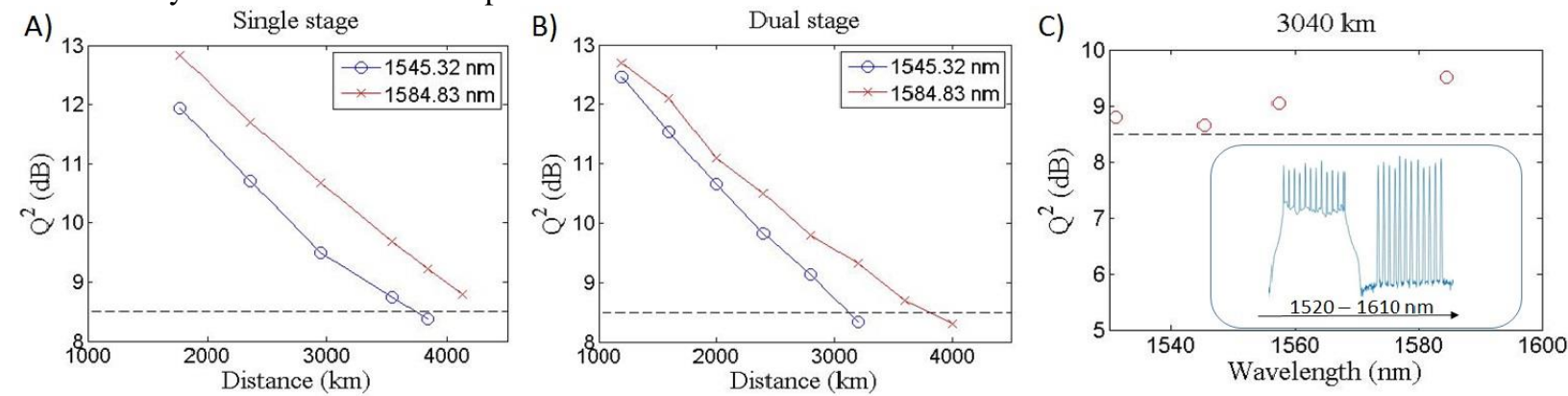

Fig. 3. Transmission results for: a) single stage discrete Raman vs distance, b) dual stage discrete Raman vs distance, c) dual stage discrete Raman for selected channels @3040 km and output spectrum in inset.

\section{Conclusions}

We have demonstrated a dual stage IDF/SMF design of discrete broadband Raman amplifier for use in a broadband $\mathrm{WDM} /$ metro network. The $5 \mathrm{~dB}$ extra amplification provided by second stage enabled us to extend the transmission span length from 59 to $80 \mathrm{~km}$. A total gain of $19.5 \mathrm{~dB}$ and $<2.5 \mathrm{~dB}$ gain flatness over $70 \mathrm{~nm}$ was achieved, and no gain equalizing elements inside the amplifier were used. The noise figure varied from $7.2 \mathrm{~dB}$ to $5.7 \mathrm{~dB}$, decreasing with wavelength. The amplifier was tested in a recirculating loop, compensating for $38 \times 80 \mathrm{~km}$ recirculation spans. Assuming an "error free" $\mathrm{Q}^{2}$ threshold of $8.5 \mathrm{~dB}$ a distance of $3040 \mathrm{~km}$ was reached for broadband transmission of $120 \mathrm{~Gb} / \mathrm{s}$ DP-QPSK signals.

\section{Acknowledgements}

This work was supported by the EPSRC project EP/M009092/1, FP7 ITN ICONE (No. 608099) and II-VI Inc.

\section{References}

[1] M. N. Islam, "Raman amplifiers for telecommunications," in IEEE J. Sel. Topics Quantum Electron., vol. 8, no. 3, pp. 548-559 (2002).

[2] S. Namiki and Y. Emori, "Ultrabroad-band Raman amplifiers pumped and gain-equalized by wavelength-division-multiplexed high-power laser diodes," in IEEE J. Sel. Topics Quantum Electron, vol. 7, no. 1, pp. 3-16 (2001).

[3] Y. Emori and S. Namiki, "100 nm bandwidth flat gain Raman amplifiers pumped and gain-equalized by 12-wavelength-channel WDM high power laser diodes," OFC/IOOC Technical Digest. Optical Fiber Communication Conference, San Diego, CA, USA, pp. PD19/1-PD19/3 (1999). [4] L. E. Nelson et al., "All-Raman-Amplified, $73 \mathrm{~nm}$ Seamless Band Transmission of 9 Tb/s Over $6000 \mathrm{~km}$ of Fiber," in IEEE Photon. Technol. Lett., vol. 26, no. 3, pp. 242-245 (2014).

[5] M. A. Iqbal et al., "Performance characterization of high gain, high output power and low noise cascaded broadband discrete Raman amplifiers," 2017 19th International Conference on Transparent Optical Networks (ICTON), Girona, Spain, 2017, pp. 1-4 (2017).

[6] S. A. E. Lewis et al., "Characterization of double Rayleigh scatter noise in Raman amplifiers," in IEEE Photon. Technol. Lett., vol. 12, no. 5, pp. 528-530 (2000).

[7] M. E. McCarthy et al., "High spectral efficiency transmission emulation for non-linear transmission performance estimation for high order modulation formats," 2014 The European Conference on Optical Communication (ECOC), Cannes, 2014, pp. 1-3 (2014).

[8] I. D. Phillips et al., "Exceeding the nonlinear-Shannon limit using Raman laser based amplification and optical phase conjugation," $O F C$ 2014, San Francisco, CA, 2014, pp. 1-3 (2014).

[9] G. Khanna et al., "Single-Carrier 400G 64QAM and 128QAM DWDM Field Trial Transmission Over Metro Legacy Links," in IEEE Photon. Technol. Lett., vol. 29, no. 2, pp. 189-192 (2017).

[10] J. Renaudier et al., "First 100-nm Continuous-Band WDM Transmission System with 115Tb/s Transport over 100km Using Novel UltraWideband Semiconductor Optical Amplifiers," 43 ${ }^{\text {rd }}$ European Conference on Optical Communication (ECOC), Gothenburg, Sweden (2017).

[11] S. Koenig et al., "Amplification of advanced modulation formats with a semiconductor optical amplifier cascade," Opt. Express 22, 1785417871 (2014). 